\title{
A priori error estimates for higher order variational discretization and mixed finite element methods of optimal control problems
}

\author{
Zuliang $\mathrm{Lu}^{1,2^{*}}$, Yanping Chen ${ }^{3}$ and Yunqing Huang ${ }^{4}$
}

\author{
* Correspondence: zulianglux@126. \\ com \\ ${ }^{1}$ College of Civil Engineering and \\ Mechanics, Xiangtan University, \\ Xiangtan 411105, PR China \\ Full list of author information is \\ available at the end of the article
}

\begin{abstract}
In this article, we investigate a priori error estimates for the optimal control problems governed by elliptic equations using higher order variational discretization and mixed finite element methods. The state and the co-state are approximated by the order $k$ Raviart-Thomas mixed finite element spaces and the control is not discreted. A priori error estimates for the higher order variational discretization and mixed finite element approximation of control problems are obtained. Finally, we present some numerical examples which confirm our theoretical results.

Mathematics Subject Classification 1991: 49J20; 65N30.
\end{abstract}

Keywords: optimal control problems, variational discretization, mixed finite element methods, a priori error estimates

\section{Introduction}

Optimal control problems governed by elliptic equations are important problems in engineering applications. Efficient numerical methods are critical for successful applications of optimal control problems in such cases. Recently, the finite element method of optimal control problems plays an important role in numerical methods for these problems. Systematic introduction of the finite element method for optimal control problems can be found in, for example, [1]. The finite element approximation of optimal control problem by piecewise constant functions is well investigated by Falk [2] and Geveci [3]. Arada et al. [4] discussed the discretization for semilinear elliptic optimal control problems. In [5], Malanowski discussed a constrained parabolic optimal control problems.

In many control problems, the objective functional contains gradient of the state variables. Thus accuracy of gradient is important in numerical approximation of the state equations. In the finite element community, mixed finite element methods should be used for discretization of the state equations in such cases. In computational optimal control problems, mixed finite element methods are not widely used in engineering simulations. In particular there doesn't seem to exist much work on theoretical analysis of mixed finite element approximation of optimal control problems in the literature. More recently, we have done some preliminary work on sharp a posteriori error estimates, error estimates and superconvergence of mixed finite element methods for optimal control problems (see, for example, [6-13]).

(c) 2012 Lu et al; licensee Springer. This is an Open Access article distributed under the terms of the Creative Commons Attribution License (http://creativecommons.org/licenses/by/2.0), which permits unrestricted use, distribution, and reproduction in any medium, provided the original work is properly cited. 
In [14], the author first presents the variational discretization concept for optimal control problems with control constraints, which implicitly utilizes the first order optimality conditions and the discretization of the state and adjoint equations for the discretization of the control instead of discretizing the space of admissible controls.

For $1 \leq p<\infty$ and $m$, any nonnegative integer, let $W^{m, p}(\Omega)=\left\{v \in L^{p}(\Omega) ; D^{\beta} v \in L^{p}\right.$ $(\Omega)$ if $|\beta| \leq m\}$ denote the Sobolev spaces endowed with the norm $\|v\|_{m, p}^{p}=\sum_{|\beta| \leq m}\left\|D^{\beta} v\right\|_{L^{p}(\Omega)}^{p}$, and the semi-norm $|v|_{m, p}^{p}=\sum_{|\beta|=m}\left\|D^{\beta} v\right\|_{L^{p}(\Omega)}^{p}$. We set $W_{0}^{m, p}(\Omega)=\left\{v \in W^{m, p}(\Omega):\left.v\right|_{\partial \Omega}=0\right\}$. For $p=2$, we denote $H^{m}(\Omega)=W^{m, 2}(\Omega), H_{0}^{m}(\Omega)=W_{0}^{m, 2}(\Omega)$, and $\|\cdot\|_{m}=\|\cdot\|_{m, 2},\|\cdot\|=\|\cdot\|_{0,2}$.

In this article we derive a priori error estimates for higher order variational discretization and mixed finite element methods of quadratic optimal control problems.

We consider the following quadratic optimal control problems:

$$
\min _{u \in K \subset U}\left\{\frac{1}{2}\left\|\boldsymbol{p}-\boldsymbol{p}_{d}\right\|^{2}+\frac{1}{2}\left\|y-y_{d}\right\|^{2}+\frac{1}{2}\|u\|^{2}\right\}
$$

subject to the state equation

$$
\begin{aligned}
& \operatorname{div} p=f+B u, \quad x \in \Omega, \\
& p=-A \nabla y, \quad x \in \Omega, \\
& y=0, \quad x \in \partial \Omega,
\end{aligned}
$$

where the bounded open set $\Omega \subset \mathbb{R}^{2}$, is a convex domain with the boundary $\partial \Omega$. We shall assume that $\boldsymbol{p}_{d} \in L^{2}(\Omega)^{2}, y_{d} \in L^{2}(\Omega), f \in H^{1}(\Omega)$, and $B$ is a continuous linear operator from $U=L^{2}(\Omega)$ to $H^{1}(\Omega)$. Furthermore, we assume the coefficient matrix $A$ $(x)=\left(a_{i, j}(x)\right)_{2 \times 2} \in\left(W^{1, \infty}(\Omega)\right)^{2 \times 2}$ is a symmetric $2 \times 2$-matrix and there is a constant $c>0$ satisfying for any vector $\mathrm{X} \in \mathbb{R}^{2}, \mathrm{X}^{\prime} A \mathrm{X} \geq c\|\mathrm{X}\|_{\mathbb{R}^{2}}^{2}$. Here, $K$ denotes the admissible set of the control variable, defined by

$$
K=\left\{u \in U=L^{2}(\Omega): \int_{\Omega} u \geq 0\right\} .
$$

Optimal control problems have been so widely met in all kinds of practical problems. Now we mention their application of the optimal control problems (1.1)-(1.4). Let us recall the static temperature control problem. Let $y$ be the temperature distribution in the body $\Omega$, which satisfies the elliptic Equations (1.2) and (1.3), where $A$ is conductivity matrix and $B u$ represents heat resource density inside the body, which can be controlled by $u$. For example, often $(B u)(x)=c(x) u(x)$, where $c(x)$ is the density factor. Assume that the body's surface temperature is fixed, say, zero. The aim of the control is to make the temperature distribution $y$ as close as to the desirable distribution $y_{d}$, e. g., $y_{d}=10$. Of course there could be many controls to achieve this objective, but we wish to achieve this using as small as possible energy, which can be represented by the formula $\int_{\Omega} u^{2}$. Then we give the temperature control model for the problem (1.1)(1.4). 
Now, we introduce the co-state elliptic equation

$$
-\operatorname{div}\left(A\left(\nabla z+\boldsymbol{p}-\boldsymbol{p}_{d}\right)\right)=y-y_{d}, \quad x \in \Omega,
$$

with the boundary condition

$$
z=0, \quad x \in \partial \Omega \text {. }
$$

The outline of this article is as follows. In Section 2, we construct the higher order variational discretization and mixed finite element approximation for optimal control problems governed by elliptic equations. Furthermore, we briefly state the definitions and properties of some interpolation operators. In Section 3, we derive a priori error estimates for the higher order variational discretization and mixed finite element solutions of the optimal control problems. Numerical examples are presented in Section 4. Finally, we analyze the conclusion and the future studies in Section 5.

\section{Variational discretization and mixed finite element methods}

We shall now describe the variational discretization and mixed finite element approximation of the optimal control problems (1.1)-(1.4). Let

$$
V=H(\operatorname{div} ; \Omega)=\left\{\boldsymbol{v} \in\left(L^{2}(\Omega)\right)^{2}, \operatorname{div} \boldsymbol{v} \in L^{2}(\Omega)\right\}, \quad W=L^{2}(\Omega) .
$$

The Hilbert space $V$ is equipped with the following norm:

$$
\|\boldsymbol{v}\|_{\text {div }}=\|\boldsymbol{v}\|_{H(\operatorname{div} ; \Omega)}=\left(\|\boldsymbol{v}\|_{0, \Omega}^{2}+\|\operatorname{div} \boldsymbol{v}\|_{0, \Omega}^{2}\right)^{1 / 2} .
$$

We recast (1.1)-(1.4) as the following weak form: find $(\boldsymbol{p}, y, u) \in V \times W \times U$ such that

$$
\begin{aligned}
& \min _{u \in K \subset U}\left\{\frac{1}{2}\left\|\boldsymbol{p}-\boldsymbol{p}_{d}\right\|^{2}+\frac{1}{2}\left\|y-y_{d}\right\|^{2}+\frac{1}{2}\|u\|^{2}\right\} \\
& \left(A^{-1} \boldsymbol{p}, \boldsymbol{v}\right)-(y, \operatorname{div} \boldsymbol{v})=0, \quad \forall \boldsymbol{v} \in V, \\
& (\operatorname{div} \boldsymbol{p}, w)=(f+B u, w), \quad \forall w \in W .
\end{aligned}
$$

It is well known (see e.g., $[15,16])$ that the optimal control problem (2.1)-(2.3) has a solution $(\boldsymbol{p}, y, u)$, and that a triplet $(\boldsymbol{p}, y, u)$ is the solution of (2.1)-(2.3) if and only if there is a co-state $(\boldsymbol{q}, z) \in V \times W$ such that $(\boldsymbol{p}, \boldsymbol{y}, \boldsymbol{q}, z, u)$ satisfies the following optimality conditions:

$$
\begin{aligned}
& \left(A^{-1} \boldsymbol{p}, \boldsymbol{v}\right)-(y, \operatorname{div} \boldsymbol{v})=0, \quad \forall \boldsymbol{v} \in V \\
& (\operatorname{div} \boldsymbol{p}, w)=(f+B u, w), \quad \forall w \in W, \\
& \left(A^{-1} \boldsymbol{q}, \boldsymbol{v}\right)-(z, \operatorname{div} \boldsymbol{v})=-\left(\boldsymbol{p}-\boldsymbol{p}_{d}, \boldsymbol{v}\right), \quad \forall \boldsymbol{v} \in V, \\
& (\operatorname{div} \boldsymbol{q}, w)=\left(y-y_{d}, w\right), \quad \forall w \in W, \\
& \left(u+B^{*} z, \tilde{u}-u\right)_{U} \geq 0, \quad \forall \tilde{u} \in K,
\end{aligned}
$$


where $(\cdot, \cdot)_{U}$ is the inner product of $U, B^{*}$ is the adjoint operator of $B$. In the rest of the article, we shall simply write the product as (., .) whenever no confusion should be caused.

Now, we will show that the control variable of the optimal control problem (2.4)(2.8) can be infinitely smooth if the special constraint set $K$ defined as (1.5).

Lemma 2.1. Let $(\boldsymbol{p}, \boldsymbol{y}, \boldsymbol{q}, z, u) \in(\boldsymbol{V} \times W)^{2} \times K$ be the solution of (2.4)-(2.8). Then we have

$$
u=\max \left(0, \overline{B^{*} z}\right)-B^{*} z,
$$

where $\overline{B^{*} z}=\int_{\Omega} B^{*} z /|\Omega|$ denotes the integral average on $\Omega$ of the function $z$.

Proof. If $\overline{B^{*} z}>0$, then $u=\overline{B^{*} z}-B^{*} z$ and for any $v \in K$

$$
\begin{aligned}
\left(u+B^{*} z, v-u\right) & =\int_{\Omega}\left(u+B^{*} z\right)(v-u) \\
& =\int_{\Omega} \overline{B^{*} z}\left(v-\overline{B^{*} z}+B^{*} z\right) \\
& =\overline{B^{*} z} \int_{\Omega} v \geq 0 .
\end{aligned}
$$

If $\overline{B^{*} z} \leq 0$, then $u=-B^{* *} z$ and $\left(u+B^{* *} z, v-u\right)=0$. Now, for the costate solution $z$, since the solution of (2.8) is unique, then we have proved the Lemma.

From Lemma 3.1, we obtain the following regularity result for the control variable.

Lemma 2.2. Let $(\boldsymbol{p}, \boldsymbol{y}, \boldsymbol{q}, z, u) \in(\boldsymbol{V} \times W)^{2} \times K$ be the solution of (2.4)-(2.8). Assume that the data functions $f, y_{d}, \boldsymbol{p}_{d}$, and the domain $\Omega$ are infinitely smooth. Then the control function $u \in C^{\infty}(\bar{\Omega})$.

Proof. By applying the regularity argument of elliptic problem (1.2)-(1.3), it is clear that $y \in H^{2}(\Omega)$, so that $\boldsymbol{p} \in H^{1}(\Omega)$. It follows from the costate Equation (1.6) and the assumption of $y_{d}, \boldsymbol{p}_{d}$, we can obtain that $z \in H^{2}(\Omega)$. Using the relationship between the control and the costate $u=\max \left(0, \overline{B^{*} z}\right)-B^{*} z$, then $u \in H^{2}(\Omega)$. Thus $y \in H^{4}(\Omega)$, $\boldsymbol{p} \in H^{3}(\Omega)$. By repeating the above process, we can conclude that $u \in C^{\infty}(\bar{\Omega})$.

Let $\mathcal{T}_{h}$ be regular triangulation of $\Omega$. They are assumed to satisassociated with the triangulationfy the angle condition which means that there is a positive constant $C$ such that for all $T \in \mathcal{T}_{h}, C^{-1} h_{T}^{2} \leq|T| \leq C h_{T}^{2}$, where $|T|$ is the area of $T, h_{T}$ is the diameter of $T$ and $h=\max h_{T}$. In addition $C$ or $c$ denotes a general positive constant independent of $h$.

Let $V_{h} \times W_{h} \subset V \times W$ denotes the Raviart-Thomas space [17] of the lowest order associated with the triangulation $\mathcal{T}_{h}$ of $\Omega . P_{k}$ denotes the space of polynomials of total degree at most $k$. Let $V(T)=\left\{v \in P_{k}^{2}(T)+x \cdot P_{k}(T)\right\}, W(T)=P_{k}(T)$. We define

$$
\begin{aligned}
& V_{h}:=\left\{\boldsymbol{v}_{h} \in V: \forall T \in \mathcal{T}_{h},\left.\boldsymbol{v}_{h}\right|_{T} \in V(T)\right\}, \\
& W_{h}:=\left\{w_{h} \in W: \forall T \in \mathcal{T}_{h},\left.w_{h}\right|_{T} \in W(T)\right\} .
\end{aligned}
$$

By the definition of finite element subspace, the mixed finite element discretization of (2.1)-(2.3) is as follows: compute $\left(\boldsymbol{p}_{h}, y_{h}, u_{h}\right) \in V_{h} \times W_{h} \times K$ such that 


$$
\begin{aligned}
& \min _{u_{h} \in K}\left\{\frac{1}{2}\left\|\boldsymbol{p}_{h}-\boldsymbol{p}_{d}\right\|^{2}+\frac{1}{2}\left\|y_{h}-y_{d}\right\|^{2}+\frac{1}{2}\left\|u_{h}\right\|^{2}\right\} \\
& \left(A^{-1} \boldsymbol{p}_{h^{\prime}} \boldsymbol{v}_{h}\right)-\left(y_{h}, \operatorname{div} \boldsymbol{v}_{h}\right)=0, \quad \forall \boldsymbol{v}_{h} \in V_{h \prime} \\
& \left(\operatorname{div} \boldsymbol{p}_{h^{\prime}} w_{h}\right)=\left(f+B u_{h}, w_{h}\right), \quad \forall w_{h} \in W_{h} .
\end{aligned}
$$

It is well known that the optimal control problem (2.10)-(2.12) again has a solution $\left(\boldsymbol{p}_{h}, y_{h}, u_{h}\right)$, and that a triplet $\left(\boldsymbol{p}_{h}, y_{h}, u_{h}\right)$ is the solution of (2.10)-(2.12) if and only if there is a co-state $\left(\boldsymbol{q}_{h}, z_{h}\right) \in \boldsymbol{V}_{h} \times W_{h}$ such that $\left(\boldsymbol{p}_{h}, y_{h}, \boldsymbol{q}_{h}, z_{h}, u_{h}\right)$ satisfies the following optimality conditions:

$$
\begin{aligned}
& \left(A^{-1} \boldsymbol{p}_{h}, \boldsymbol{v}_{h}\right)-\left(y_{h}, \operatorname{div} \boldsymbol{v}_{h}\right)=0, \quad \forall \boldsymbol{v}_{h} \in V_{h \prime} \\
& \left(\operatorname{div} \boldsymbol{p}_{h}, w_{h}\right)=\left(f+B u_{h}, w_{h}\right), \quad \forall w_{h} \in W_{h}, \\
& \left(A^{-1} \boldsymbol{q}_{h}, \boldsymbol{v}_{h}\right)-\left(z_{h}, \operatorname{div} \boldsymbol{v}_{h}\right)=-\left(\boldsymbol{p}_{h}-\boldsymbol{p}_{d}, \boldsymbol{v}_{h}\right) \quad \forall \boldsymbol{v}_{h} \in V_{h}, \\
& \left(\operatorname{div} \boldsymbol{q}_{h}, w_{h}\right)=\left(y_{h}-y_{d}, w_{h}\right), \quad \forall w_{h} \in W_{h \prime} \\
& \left(u_{h}+B^{*} z_{h}, \tilde{u}-u_{h}\right) \geq 0, \quad \forall \tilde{u} \in K .
\end{aligned}
$$

Let $P_{h}: W \rightarrow W_{h}$ be the orthogonal $L^{2}(\Omega)$-projection into $W_{h}$ define by [18]:

$$
\left(P_{h} w-w, \mathcal{X}\right)=0, \quad w \in W, \quad \mathcal{X} \in W_{h}
$$

which satisfies

$$
\begin{aligned}
& \left\|P_{h} w-w\right\|_{0, q} \leq C\|w\|_{t, q} h^{t}, \quad 0 \leq t \leq k+1, \quad \text { if } w \in W \cap W^{t, q}(\Omega), \\
& \left\|P_{h} w-w\right\|_{-r} \leq C\|w\|_{t} h^{r+t}, \quad 0 \leq r, \quad t \leq k+1, \quad \text { if } w \in H^{t}(\Omega) \\
& \left(\operatorname{div} v_{h}, w-P_{h} w\right)=0, \quad w \in W, \quad v_{h} \in V_{h} .
\end{aligned}
$$

Let $\pi_{h}: V \rightarrow V_{h}$ be the Raviart-Thomas projection [19], which satisfies

$$
\begin{aligned}
& \left(\operatorname{div}\left(\pi_{h} v-v\right), w_{h}\right)=0, \quad v \in V, \quad w \in W_{h}, \\
& \left\|\pi_{h} v-v\right\|_{0, q} \leq C\|v\|_{t, q} h^{t}, \quad 1 / q<t \leq k+1, \quad \text { if } v \in V \cap W^{t, q}(\Omega)^{2}, \\
& \left\|\operatorname{div}\left(\pi_{h} v-v\right)\right\|_{0} \leq C\|\operatorname{div} v\|_{t} h^{t}, \quad 0 \leq t \leq k+1, \quad \text { if } v \in V \cap H^{t}(\operatorname{div} ; \Omega) .
\end{aligned}
$$

We have the commuting diagram property [20]

$$
\operatorname{div} \circ \pi_{h}=P_{h} \circ \operatorname{div}: V \rightarrow W_{h} \quad \text { and } \quad \operatorname{div}\left(I-\pi_{h}\right) V \perp W_{h},
$$

where and after, $I$ denotes identity matrix. 


\section{A priori error estimates}

In the rest of the article, we shall use some intermediate variables. For any control function $\tilde{u} \in K$, we first define the state solution $(\boldsymbol{p}(\tilde{u}), y(\tilde{u}), \boldsymbol{q}(\tilde{u}), z(\tilde{u}))$ associated with $\tilde{u}$ that satisfies

$$
\begin{aligned}
& \left(A^{-1} \boldsymbol{p}(\tilde{u}), \boldsymbol{v}\right)-(\gamma(\tilde{u}), \operatorname{div} \boldsymbol{v})=0, \quad \forall \boldsymbol{v} \in V, \\
& (\operatorname{div} p(\tilde{u}), w)=(f+B \tilde{u}, w), \quad \forall w \in W, \\
& \left(A^{-1} \boldsymbol{q}(\tilde{u}), v\right)-(z(\tilde{u}), \operatorname{div} v)=-\left(\boldsymbol{p}(\tilde{u})-\boldsymbol{p}_{d^{\prime}} \boldsymbol{v}\right), \quad \forall \boldsymbol{v} \in V, \\
& (\operatorname{div} \boldsymbol{q}(\tilde{u}), w)=\left(y(\tilde{u})-\gamma_{d}, w\right), \quad \forall w \in W .
\end{aligned}
$$

Correspondingly, we define the discrete state solution $\left(\boldsymbol{p}_{h}(\tilde{u}), y_{h}(\tilde{u}), \boldsymbol{q}_{h}(\tilde{u}), z_{h}(\tilde{u})\right)$ associated with $\tilde{u} \in K$ that satisfies

$$
\begin{aligned}
& \left(A^{-1} \boldsymbol{p}_{h}(\tilde{u}), \boldsymbol{v}_{h}\right)-\left(y_{h}(\tilde{u}), \operatorname{div} \boldsymbol{v}_{h}\right)=0, \quad \forall \boldsymbol{v}_{h} \in V_{h}, \\
& \left(\operatorname{div} \boldsymbol{p}_{h}(\tilde{u}), w_{h}\right)=\left(f+B \tilde{u}, w_{h}\right), \quad \forall w_{h} \in W_{h}, \\
& \left(A^{-1} \boldsymbol{q}_{h}(\tilde{u}), \boldsymbol{v}_{h}\right)-\left(z_{h}(\tilde{u}), \operatorname{div} \boldsymbol{v}_{h}\right)=-\left(\boldsymbol{p}_{h}(\tilde{u})-\boldsymbol{p}_{d}, \boldsymbol{v}_{h}\right), \quad \forall v_{h} \in V_{h}, \\
& \left(\operatorname{div} \boldsymbol{q}_{h}(\tilde{u}), w_{h}\right)=\left(y_{h}(\tilde{u})-y_{d}, w_{h}\right), \quad \forall w_{h} \in W_{h} .
\end{aligned}
$$

We define another discrete state solution $\left(\hat{\boldsymbol{p}}_{h}(u), \hat{z}_{h}(u)\right)$ that satisfies

$$
\begin{aligned}
& \left(A^{-1} \hat{\boldsymbol{q}}_{h}(\tilde{u}), \boldsymbol{v}_{h}\right)-\left(\hat{z}_{h}(\tilde{u}), \operatorname{div} \boldsymbol{v}_{h}\right)=-\left(\boldsymbol{p}-\boldsymbol{p}_{d}, \boldsymbol{v}_{h}\right), \quad \forall \boldsymbol{v}_{h} \in V_{h}, \\
& \left(\operatorname{div} \hat{\boldsymbol{q}}_{h}(\tilde{u}), w_{h}\right)=\left(y-y_{d}, w_{h}\right), \quad \forall w_{h} \in W_{h} .
\end{aligned}
$$

Thus, as we defined, the exact solution and its approximation can be written in the following way:

$$
\begin{aligned}
\left(\boldsymbol{p}, y_{1} \boldsymbol{q}, z\right) & =(\boldsymbol{p}(u), y(u), \boldsymbol{q}(u), z(u)), \\
\left(\boldsymbol{p}_{h^{\prime}} y_{h}, \boldsymbol{q}_{h^{\prime}}, z_{h}\right) & =\left(\boldsymbol{p}_{h}\left(u_{h}\right), y_{h}\left(u_{h}\right), \boldsymbol{q}_{h}\left(u_{h}\right), z_{h}\left(u_{h}\right)\right) .
\end{aligned}
$$

Combining Lemma 2.1 in [19] and (2.19), we obtain the following technical results:

Lemma 3.1. Let $\omega \in V, \phi \in L^{2}(\Omega)^{2}$, and $\psi \in L^{2}(\Omega)$. If $\tau \in W_{h}$ satisfies

$$
\begin{cases}\left(A^{-1} \boldsymbol{\omega}, \boldsymbol{v}_{h}\right)-\left(\tau, \operatorname{div} \boldsymbol{v}_{h}\right)=\left(\boldsymbol{\varphi}, v_{h}\right), & \forall v_{h} \in V_{h} \\ \left(\operatorname{div} \boldsymbol{\omega}, w_{h}\right)=\left(\psi, w_{h}\right), & \forall w_{h} \in W_{h},\end{cases}
$$

then, there exists a constant $C$ such that

$$
\|\tau\|_{0} \leq C\left(h\|\omega\|_{0}+h\|\operatorname{div} \omega\|_{0}+\|\varphi\|_{0}+\|\psi\|_{0}\right),
$$

for h sufficiently small.

Now we chose $\tilde{u}=u$ in (3.5)-(3.8), then we set some intermediate errors:

$$
\varepsilon_{1}:=\boldsymbol{p}-\boldsymbol{p}_{h}(u) \text { and } e_{1}:=\gamma-y_{h}(u) .
$$


To analyze the intermediate errors, let us first note the following error equations from (2.4), (2.5), (3.5) and (3.6) with the choice $\tilde{u}=u$ :

$$
\begin{aligned}
& \left(A^{-1} \varepsilon_{1}, v_{h}\right)-\left(e_{1}, \operatorname{div} v_{h}\right)=0, \quad \forall v_{h} \in V_{h}, \\
& \left(\operatorname{div} \varepsilon_{1}, w_{h}\right)=0, \quad \forall w_{h} \in W_{h} .
\end{aligned}
$$

By (2.18)-(2.24) and Lemma 3.1, we can establish the following error estimates:

Theorem 3.1. Assume that $y \in H^{k+3}(\Omega)$. If $h$ is sufficiently small, there is a positive constant $C$ independent of $h$ such that

$$
\begin{gathered}
\left\|y-y_{h}(u)\right\|_{0} \leq C h^{k+1}, \\
\left\|\boldsymbol{p}-\boldsymbol{p}_{h}(u)\right\|_{0} \leq C h^{k+1}, \\
\left\|\boldsymbol{p}-\boldsymbol{p}_{h}(u)\right\|_{\text {div }} \leq C h^{k+1} .
\end{gathered}
$$

Proof. Let $\tau=P_{h} y-y_{h}(u)$ and $\sigma=\pi_{h} \boldsymbol{p}-\boldsymbol{p}_{h}(u)$. Rewrite (3.13) and (3.14) in the form

$$
\left(A^{-1} \varepsilon_{1}, \boldsymbol{v}_{h}\right)-\left(\tau, \operatorname{div} \boldsymbol{v}_{h}\right)=0, \quad \forall \boldsymbol{v}_{h} \in V_{h}
$$

$$
\left(\operatorname{div} \varepsilon_{1}, w_{h}\right)=0, \quad \forall w_{h} \in W_{h} .
$$

It follows from Lemma 3.1 that

$$
\|\tau\|_{0} \leq C\left(h\left\|\varepsilon_{1}\right\|_{0}+h\left\|\operatorname{div} \varepsilon_{1}\right\|_{0}\right) .
$$

By using (2.19) that

$$
\begin{aligned}
\left\|e_{1}\right\|_{0} & =\left\|y-y_{h}(u)\right\|_{0} \\
& =\|P h y-y\|_{0}+\|\tau\|_{0} \\
& \leq C\left(h\left\|\varepsilon_{1}\right\|_{0}+h\left\|\operatorname{div} \varepsilon_{1}\right\|_{0}+h^{k+1}\|y\|_{k+1}\right) .
\end{aligned}
$$

If we now again rewrite (3.13) and (3.14) as

$$
\begin{aligned}
& \left(A^{-1} \sigma, \boldsymbol{v}_{h}\right)-\left(\tau, \operatorname{div} \boldsymbol{v}_{h}\right)=\left(A^{-1}\left(\pi_{h} \boldsymbol{p}-\boldsymbol{p}\right), \boldsymbol{v}_{h}\right), \quad \forall \boldsymbol{v}_{h} \in V_{h}, \\
& \left(\operatorname{div} \sigma, w_{h}\right)=0, \quad \forall w_{h} \in W_{h} .
\end{aligned}
$$

Using the standard stability results of mixed finite element methods in [21], we establish the following results:

$$
\begin{aligned}
\|\sigma\|_{\text {div }} & \leq C\left(\left\|\pi_{h} p-p\right\|_{0}+\left\|e_{1}\right\|_{0}\right) \\
& \leq C\left(h^{k+1}\|y\|_{k+2}+\left\|e_{1}\right\|_{0}\right) .
\end{aligned}
$$

From (3.24), (2.23), and the commuting diagram property (2.25) we now obtain the bounds 


$$
\begin{aligned}
\left\|\varepsilon_{1}\right\|_{0} & \leq C\left(\left\|\pi_{h} \boldsymbol{p}-\boldsymbol{p}\right\|_{0}+\|\sigma\|_{0}\right) \\
& \leq C\left(h^{k+1}\|y\|_{k+2}+\left\|e_{1}\right\|_{0}\right)
\end{aligned}
$$

and

$$
\begin{aligned}
\left\|\operatorname{div} \varepsilon_{1}\right\|_{0} & \leq C\left(\left\|\operatorname{div}\left(\pi_{h} p-p\right)\right\|_{0}+\|\operatorname{div} \sigma\|_{0}\right) \\
& =C\left(\left\|P_{h} \circ \operatorname{div} \boldsymbol{p}-\operatorname{div} \boldsymbol{p}\right\|_{0}+\|\operatorname{div} \sigma\|_{0}\right) \\
& \leq C\left(h^{k+1}\|y\|_{k+3}+\left\|e_{1}\right\| 0\right),
\end{aligned}
$$

when substituted into (3.21), which yields the estimate

$$
\left\|e_{1}\right\|_{0} \leq C\left(h\left\|e_{1}\right\|_{0}+h^{k+1}\|y\|_{k+1}\right) \text {. }
$$

Then (3.27) implies (3.15) holds if $h$ is small enough. Applying (3.27) to (3.25) and (3.26) shows that (3.16) and (3.17) also hold.

With the intermediate errors, we decompose the errors as follows

$$
\begin{aligned}
& \boldsymbol{p}-\boldsymbol{p}_{h}=\boldsymbol{p}-\boldsymbol{p}_{h}(u)+\boldsymbol{p}_{h}(u)-\boldsymbol{p}_{h}:=\varepsilon_{1}+\epsilon_{1}, \\
& y-y_{h}=y-y_{h}(u)+y_{h}(u)-y_{h}:=e_{1}+r_{1} .
\end{aligned}
$$

From (2.13), (2.14), (3.5) and (3.6), we have

$$
\begin{aligned}
& \left(A^{-1} \epsilon_{1}, v_{h}\right)-\left(r_{1}, \operatorname{div} v_{h}\right)=0, \quad \forall v_{h} \in V_{h} \\
& \left(\operatorname{div} \epsilon_{1}, w_{h}\right)=\left(B\left(u-u_{h}\right), w_{h}\right), \quad \forall w_{h} \in W_{h} .
\end{aligned}
$$

The assumption that $A \in L^{\infty}\left(\Omega ; \mathbb{R}^{2 \times 2}\right)$ implies that it is bounded that the inverse operator of the map $\left\{\epsilon_{1}, r_{1}\right\}: \mathbb{R}^{3} \rightarrow V \times W$ defined by the above saddle-point problem [21]:

$$
\left\|\epsilon_{1}\right\|_{\text {div }}+\left\|r_{1}\right\|_{0} \leq C\left\|u-u_{h}\right\|_{0},
$$

where the continuity of the linear operator $B$ has been used.

Now we are able to derive our main results.

Theorem 3.2. Let $(\boldsymbol{p}, y, \boldsymbol{q}, z, u) \in(\boldsymbol{V} \times W)^{2} \times K$ and $\left(\boldsymbol{p}_{h}, y_{h}, \boldsymbol{q}_{h}, z_{h}, u_{h}\right) \in\left(V_{h} \times W_{h}\right)^{2}$ $\times K$ be the solutions of(2.4)-(2.8) and (2.13)-(2.17), respectively. We assume thaty, $z \in$ $H^{k+3}(\Omega)$.

Then, we have

$$
\begin{aligned}
& \left\|u-u_{h}\right\| 0 \leq C h^{k+1}, \\
& \left\|\boldsymbol{p}-\boldsymbol{p}_{h}\right\|_{\text {div }}+\left\|y-y_{h}\right\|_{0} \leq C h^{k+1}, \\
& \left\|\boldsymbol{q}-\boldsymbol{q}_{h}\right\|_{\text {div }}+\left\|z-z_{h}\right\|_{0} \leq C h^{k+1} .
\end{aligned}
$$

Proof. We choose $\tilde{u}=u_{h}$ in (2.8) and $\tilde{u}=u$ in (2.17) to get that

$$
\left(u+B^{*} z, u_{h}-u\right) \geq 0,
$$


and

$$
\left(u_{h}+B^{*} z_{h}, u-u_{h}\right) \geq 0 .
$$

Then we have

$$
\begin{aligned}
\left\|u-u_{h}\right\|^{2} & \leq\left(B^{*} z_{h}-B^{*} z, u-u_{h}\right) \\
& =\left(B^{*} \hat{z}_{h}(u)-B^{*} z, u-u_{h}\right)+\left(B^{*} z_{h}-B^{*} \hat{z}_{h}(u), u-u_{h}\right) .
\end{aligned}
$$

Moreover, the $\delta$-Caunchy inequality leads that

$$
\begin{aligned}
\left(B^{*} \hat{z}_{h}(u)-B^{*} z, u-u_{h}\right) & \leq C\left\|\hat{z}_{h}(u)-z\right\| \cdot\left\|u-u_{h}\right\| \\
& \leq C\left\|\hat{z}_{h}(u)-z\right\|^{2}+C \delta\left\|u-u_{h}\right\|^{2}
\end{aligned}
$$

where $\delta$ is an arbitrary positive number. From (3.30)-(3.31), (2.15)-(2.16) and (3.9)(3.10), we obtain the following equations:

$$
\begin{aligned}
& \left(A^{-1}\left(\boldsymbol{p}_{h}-\boldsymbol{p}_{h}(u)\right), \boldsymbol{v}_{h}\right)-\left(y_{h}-y_{h}(u), \operatorname{div} \boldsymbol{v}_{h}\right)=0, \quad \forall \boldsymbol{v}_{h} \in V_{h \prime} \\
& \left(\operatorname{div}\left(\boldsymbol{p}_{h}-\boldsymbol{p}_{h}(u)\right), w_{h}\right)=\left(B\left(u-u_{h}\right), w_{h}\right), \quad \forall w_{h} \in W_{h \prime} \\
& \left(A^{-1}\left(\hat{\boldsymbol{q}}_{h}(u)-\boldsymbol{q}_{h}\right), \boldsymbol{v}_{h}\right)-\left(\hat{z}_{h}(u)-z_{h}, \operatorname{div} \boldsymbol{v}_{h}\right)=-\left(\boldsymbol{p}-\boldsymbol{p}_{h}, \boldsymbol{v}_{h}\right), \quad \forall \boldsymbol{v}_{h} \in V_{h \prime} \\
& \left(\operatorname{div}\left(\hat{\boldsymbol{q}}_{h}(u)-\boldsymbol{q}_{h}\right), w_{h}\right)=\left(y-y_{h}, w_{h}\right), \quad \forall w_{h} \in W_{h} .
\end{aligned}
$$

By applying the above error equations, we obtain

$$
\begin{aligned}
& \left(B^{*} z_{h}-B^{*} \hat{z}_{h}(u), u-u_{h}\right) \\
= & \left(z_{h}-\hat{z}_{h}(u), B\left(u-u_{h}\right)\right) \\
= & \left(\operatorname{div}\left(\boldsymbol{p}_{h}-\boldsymbol{p}_{h}(u)\right), z_{h}-\hat{z}_{h}(u)\right) \\
= & \left(A^{-1}\left(\hat{\boldsymbol{q}}_{h}(u)-\boldsymbol{q}_{h}\right), \boldsymbol{p}_{h}-\boldsymbol{p}_{h}(u)\right)+\left(\boldsymbol{p}-\boldsymbol{p}_{h^{\prime}} \boldsymbol{p}_{h}-\boldsymbol{p}_{h}(u)\right) \\
= & \left(y_{h}-y_{h}(u), \operatorname{div}\left(\hat{\boldsymbol{q}}_{h}(u)-\boldsymbol{q}_{h}\right)+\left(\boldsymbol{p}-\boldsymbol{p}_{h^{\prime}} \boldsymbol{p}_{h}-\boldsymbol{p}_{h}(u)\right)\right. \\
= & \left(y-y_{h}, y_{h}-y_{h}(u)\right)+\left(\boldsymbol{p}-\boldsymbol{p}_{h^{\prime}} \boldsymbol{p}_{h}-\boldsymbol{p}_{h}(u)\right) \\
= & \left(y-y_{h}, y-y_{h}(u)\right)+\left(\boldsymbol{p}-\boldsymbol{p}_{h^{\prime}} \boldsymbol{p}-\boldsymbol{p}_{h}(u)\right)-\left\|y-y_{h}\right\|^{2}-\left\|\boldsymbol{p}-\boldsymbol{p}_{h}\right\|^{2} \\
= & \left\|y-y_{h}\right\| \cdot\left\|y-y_{h}(u)\right\|+\left\|\boldsymbol{p}-\boldsymbol{p}_{h}\right\| \cdot\left\|\boldsymbol{p}-\boldsymbol{p}_{h}(u)\right\|-\left\|y-y_{h}\right\|^{2}-\left\|\boldsymbol{p}-\boldsymbol{p}_{h}\right\|^{2} \\
\leq & C\left\|y-y_{h}(u)\right\|^{2}+C\left\|\boldsymbol{p}-\boldsymbol{p}_{h}(u)\right\|^{2}-\frac{1}{2}\left\|y-y_{h}\right\|^{2}-\frac{1}{2}\left\|\boldsymbol{p}-\boldsymbol{p}_{h}\right\|^{2} .
\end{aligned}
$$

From (3.38), (3.39), and (3.44), we derive that

$$
\begin{gathered}
\left\|u-u_{h}\right\|^{2}+\left\|y-y_{h}\right\|^{2}+\left\|\boldsymbol{p}-\boldsymbol{p}_{h}\right\|^{2} \\
\leq C\left\|y-y_{h}(u)\right\|^{2}+C\left\|\boldsymbol{p}-\boldsymbol{p}_{h}(u)\right\|^{2}+C\left\|z-\hat{z}_{h}(u)\right\|^{2} .
\end{gathered}
$$

Note that $\hat{z}_{h}(u), \hat{\boldsymbol{q}}_{h}(u)$ are the mixed finite element approximation of $z, \boldsymbol{q}$, using the results of [22], we have

$$
\left\|\boldsymbol{q}-\hat{\boldsymbol{q}}_{h}(u)\right\|_{\mathrm{div}}+\left\|z-\hat{z}_{h}(u)\right\| \leq C h^{k+1} .
$$


From the Theorem 3.1, (3.45), and (3.46), we obtain

$$
\left\|u-u_{h}\right\|+\left\|y-y_{h}\right\|+\left\|\boldsymbol{p}-\boldsymbol{p}_{h}\right\| \leq C h^{k+1}
$$

then we derive (3.33). By using (3.42) and (3.43) and the stability results of mixed finite element methods [21], we have

$$
\left\|\hat{\boldsymbol{q}}_{h}(u)-\boldsymbol{q}_{h}\right\|_{\mathrm{div}}+\left\|\hat{z}_{h}(u)-z_{h}\right\| \leq C\left\|\boldsymbol{p}-\boldsymbol{p}_{h}\right\|+C\left\|y-y_{h}\right\| .
$$

Combining (3.46)-(3.48), we derive the following result

$$
\left\|\boldsymbol{q}-\boldsymbol{q}_{h}\right\|_{\mathrm{div}}+\left\|z-z_{h}\right\| \leq C h^{k+1} .
$$

From (3.17), (3.32), and (3.47), it is easy to see that

$$
\left\|\boldsymbol{p}-\boldsymbol{p}_{h}\right\|_{\mathrm{div}} \leq C h^{k+1} \text {. }
$$

Then we derive the results (3.34) and (3.35).

\section{Numerical tests}

In this section, we are going to validate the a priori error estimates for the error in the control, state, and co-state numerically. The optimization problems were dealt numerically with codes developed based on AFEPACK. The package is freely available and the details can be found at [23].

In our numerical examples, we consider the following optimal control problems:

$$
\begin{aligned}
& \min _{u \in K}\left\{\frac{1}{2}\left\|\boldsymbol{p}-\boldsymbol{p}_{d}\right\|^{2}+\frac{1}{2}\left\|y-y_{d}\right\|^{2}+\frac{1}{2}\|u\|^{2}\right\} \\
& \operatorname{div} \boldsymbol{p}=B u+f, \quad \boldsymbol{p}=-A \nabla y, \quad x \in \Omega,\left.\quad y\right|_{\partial \Omega}=0, \\
& \operatorname{div} \boldsymbol{q}=y-y_{d}, \quad \boldsymbol{q}=-A\left(\nabla z+\boldsymbol{p}-\boldsymbol{p}_{d}\right), \quad x \in \Omega,\left.\quad z\right|_{\partial \Omega}=0 .
\end{aligned}
$$

In our examples, we choose the domain $\Omega=[0,1] \times[0,1]$ and $A=B=I$. We present below two examples to illustrate the theoretical results of the optimal control problems. The convergence order is computed by the following formula: order $\simeq \frac{\log \left(E_{i} / E_{i+1}\right)}{\log \left(h_{i} / h_{i+1}\right)}$, where $i$ responds to the spatial partition, and $E_{i}$ denotes the for the state, costate and control approximation.

Example 1. In this example we set the other known functions as follows:

$$
\begin{aligned}
y & =2 \sin \pi x_{1} \sin \pi x_{2}, \\
z & =-\sin \pi x_{1} \sin \pi x_{2}, \\
\boldsymbol{q} & =\left(\pi \cos \pi x_{1} \sin \pi x_{2}, \pi \cos \pi x_{2} \sin \pi x_{1}\right), \\
y_{d} & =\left(1+\pi^{2}\right) y, \quad \boldsymbol{p}=\boldsymbol{p}_{d}=-2 \boldsymbol{q}, \\
f & =2 \pi^{2} y-u, \\
u & =\max (\bar{z}, 0)-z .
\end{aligned}
$$

In this numerical implementation, the error $\left\|u-u_{h}\right\|_{0},\left\|\boldsymbol{p}-\boldsymbol{p}_{h}\right\|_{\text {div }},\left\|y-y_{h}\right\|_{0}, \| \boldsymbol{q}-$ $\boldsymbol{q}_{h} \|_{\text {div }}$, and $\left\|z-z_{h}\right\|_{0}$ obtained on RT0 mixed finite element approximation and RT1 mixed finite element approximation for state function are presented in Tables 1, 2, 3 
Table 1 The numerical errors on RTO mixed finite element for state function

\begin{tabular}{|c|c|c|c|c|c|c|c|c|c|c|}
\hline \multirow[t]{2}{*}{$h$} & \multicolumn{10}{|c|}{ Errors } \\
\hline & $\left\|u-u_{h}\right\|$ & Rate & $\left\|p-p_{h}\right\|$ & Rate & $\left\|y-y_{h}\right\|$ & Rate & $\left\|q-q_{h}\right\|$ & Rate & $\left\|z-z_{h}\right\|$ & Rate \\
\hline $1 / 16$ & $3.63 e-02$ & - & $3.25 e-01$ & - & $7.24 e-02$ & - & $1.63 e-01$ & - & $3.63 e-02$ & - \\
\hline $1 / 32$ & $1.80 e-02$ & 1.01 & $1.62 e-01$ & 1.00 & $3.63 e-02$ & 0.99 & $8.12 e-02$ & 1.01 & $1.80 e-02$ & 1.01 \\
\hline $1 / 64$ & $9.06 e-03$ & 0.99 & $8.13 e-02$ & 0.99 & $1.78 e-02$ & 1.03 & $4.07 e-02$ & 1.00 & $9.06 e-03$ & 0.99 \\
\hline $1 / 128$ & $4.48 e-03$ & 1.01 & $4.03 e-02$ & 1.01 & $8.87 e-03$ & 1.01 & $2.05 e-02$ & 0.99 & $4.48 e-03$ & 1.01 \\
\hline
\end{tabular}

and 4. The theoretical results can be observed clearly from the data. The profile of the

Table 2 The numerical errors on RT1 mixed finite element for state function

\begin{tabular}{|c|c|c|c|c|c|c|c|c|c|c|}
\hline \multirow[t]{2}{*}{$h$} & \multicolumn{10}{|c|}{ Errors } \\
\hline & $\left\|u-u_{h}\right\|$ & Rate & $\left\|p-p_{h}\right\|$ & Rate & $\left\|y-y_{h}\right\|$ & Rate & $\left\|q-q_{h}\right\|$ & Rate & $\left\|z-z_{h}\right\|$ & Rate \\
\hline $1 / 16$ & $1.25 e-03$ & - & $7.13 e-03$ & - & $2.50 e-03$ & - & $3.53 e-03$ & - & $1.25 e-03$ & - \\
\hline $1 / 32$ & $3.13 e-04$ & 2.00 & $1.76 e-03$ & 2.02 & $6.24 e-04$ & 2.00 & $8.82 e-04$ & 2.00 & $3.13 e-04$ & 2.00 \\
\hline $1 / 64$ & $7.68 e-05$ & 2.02 & $4.43 e-04$ & 1.99 & $1.56 e-04$ & 2.00 & $2.23 e-04$ & 1.98 & $7.68 e-05$ & 2.02 \\
\hline $1 / 128$ & $1.90 e-05$ & 2.01 & $1.12 e-04$ & 1.98 & $3.89 e-05$ & 2.00 & $5.52 e-05$ & 2.01 & $1.90 e-05$ & 2.01 \\
\hline
\end{tabular}

Table 3 The numerical error on RTO mixed finite element for state function

\begin{tabular}{|c|c|c|c|c|c|c|c|c|c|c|}
\hline \multirow[t]{2}{*}{$h$} & \multicolumn{10}{|c|}{ Errors } \\
\hline & $\left\|u-u_{h}\right\|$ & Rate & $\left\|p-p_{h}\right\|$ & Rate & $\left\|y-y_{h}\right\|$ & Rate & $\left\|q-q_{h}\right\|$ & Rate & $\left\|z-z_{h}\right\|$ & Rate \\
\hline $1 / 16$ & $3.66 e-02$ & - & $1.63 e-01$ & - & $3.67 e-02$ & - & $1.63 e-01$ & - & $3.66 e-02$ & - \\
\hline $1 / 32$ & $1.79 e-02$ & 1.03 & $8.14 e-02$ & 1.00 & $1.79 e-02$ & 1.04 & $8.14 e-02$ & 1.00 & $1.79 e-02$ & 1.03 \\
\hline $1 / 64$ & $8.94 e-03$ & 0.99 & $4.09 e-02$ & 0.99 & $8.94 e-03$ & 0.99 & $4.09 e-02$ & 0.99 & $8.94 e-03$ & 0.99 \\
\hline $1 / 128$ & $4.50 e-03$ & 0.99 & $2.04 e-02$ & 1.00 & $4.46 e-03$ & 1.00 & $2.04 \mathrm{e}-02$ & 1.00 & $4.50 e-03$ & 0.99 \\
\hline
\end{tabular}

numerical solution is plotted in Figures 1 and 2.

Example 2. In this example we set the other known functions as follows:

$$
\begin{aligned}
y & =\left(x_{1}+x_{2}\right) \sin \pi x_{1} \sin \pi x_{2}, \\
z & =-\left(x_{1}+x_{2}\right) \sin \pi x_{1} \sin \pi x_{2}, \\
u & =\max (\bar{z}, 0)-z, \quad p=p_{d}=-\boldsymbol{q}, \\
f & =2 \pi^{2} \sin \pi x_{1} \sin \pi x_{2}\left(x_{1}+x_{2}\right)+\cos \pi x_{1} \sin \pi x_{2}-u, \\
\boldsymbol{q} & =\left(\pi \cos \pi x_{1} \sin \pi x_{2}+\sin \pi x_{1} \sin \pi x_{2}, \pi \cos \pi x_{2} \sin \pi x_{1}+\sin \pi x_{1} \sin \pi x_{2}\right), \\
y_{d} & =y+2 \pi^{2} \sin \pi x_{1} \cos \pi x_{2}\left(x_{1}+x_{2}\right)-2 \pi \cos \pi x_{1} \sin \pi x_{2}-2 \pi \sin \pi x_{1} \cos \pi x_{2} .
\end{aligned}
$$

\begin{tabular}{|c|c|c|c|c|c|c|c|c|c|c|}
\hline \multirow[t]{2}{*}{$h$} & \multicolumn{10}{|c|}{ Errors } \\
\hline & $\left\|u-u_{h}\right\|$ & Rate & $\left\|p-p_{h}\right\|$ & Rate & $\left\|y-y_{h}\right\|$ & Rate & $\left\|q-q_{h}\right\|$ & Rate & $\left\|z-z_{h}\right\|$ & Rate \\
\hline $1 / 16$ & $1.55 e-03$ & - & $5.68 e-03$ & - & $1.55 e-03$ & - & $5.68 e-03$ & - & $1.55 e-03$ & - \\
\hline $1 / 32$ & $3.88 e-04$ & 2.00 & $1.43 e-03$ & 2.00 & $3.88 e-04$ & 2.00 & $1.43 e-03$ & 2.00 & $3.88 e-04$ & 2.00 \\
\hline $1 / 64$ & $9.69 e-05$ & 2.00 & $3.58 e-04$ & 1.99 & $9.69 e-05$ & 2.00 & $3.58 e-04$ & 1.99 & $9.66 e-05$ & 2.00 \\
\hline $1 / 128$ & $2.43 e-05$ & 1.99 & $8.96 e-05$ & 2.00 & $2.43 e-05$ & 1.99 & $8.97 e-05$ & 2.00 & $2.42 e-05$ & 1.99 \\
\hline
\end{tabular}

The example obviously indicates that the error estimates remains in output data. From the error data in the two examples, it can be seen that the priori error estimates that we have mentioned is exact.

Table 4 The numerical error on RT1 mixed finite element for state function 


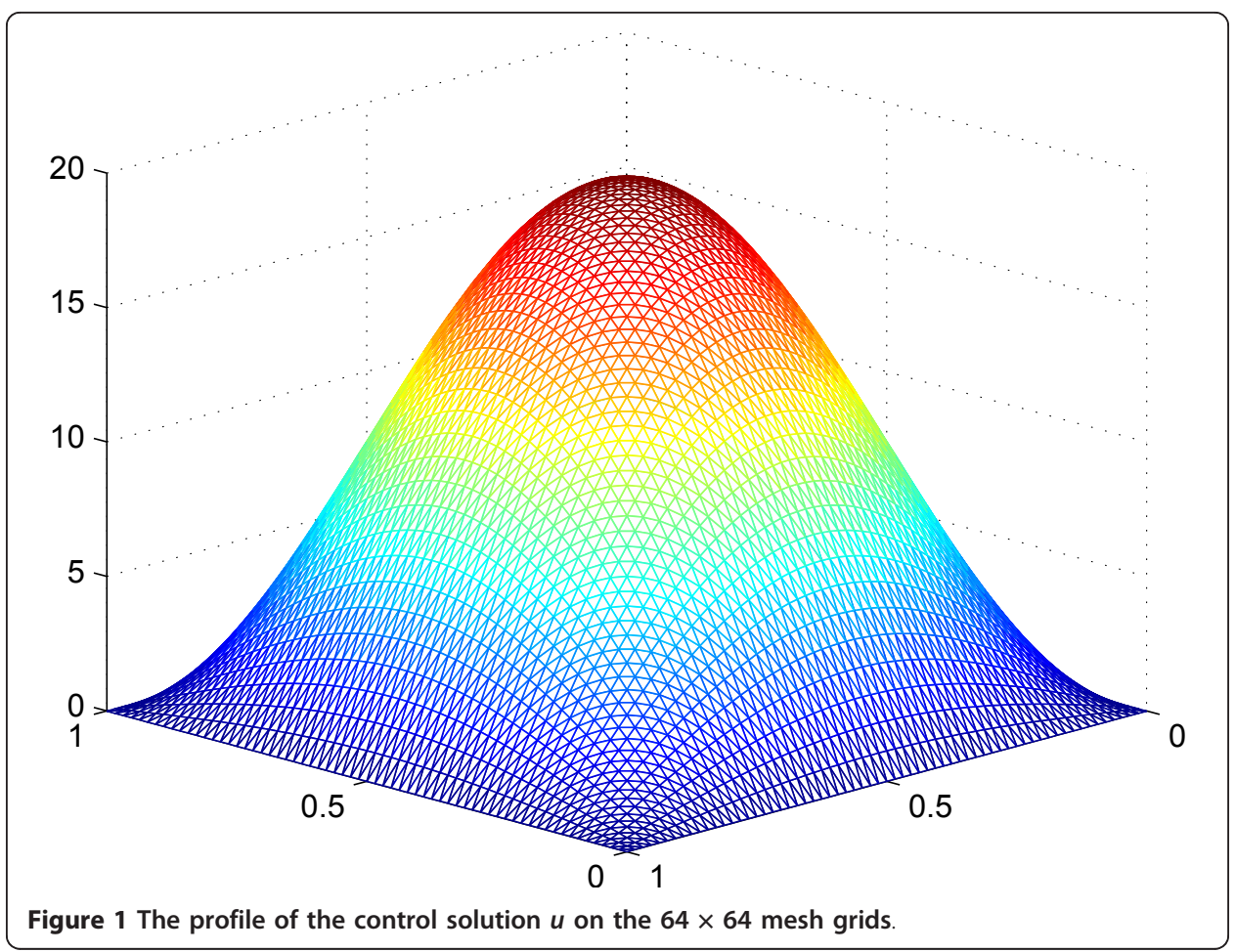

\section{Conclusion and future works}

The present article discussed the higher order variational discretization and mixed finite element methods for the optimal control problems governed by elliptic equations. We have obtained some error estimate results for both the state, the co-state and the control approximation with convergence order $h^{k+1}$. The priori error estimates

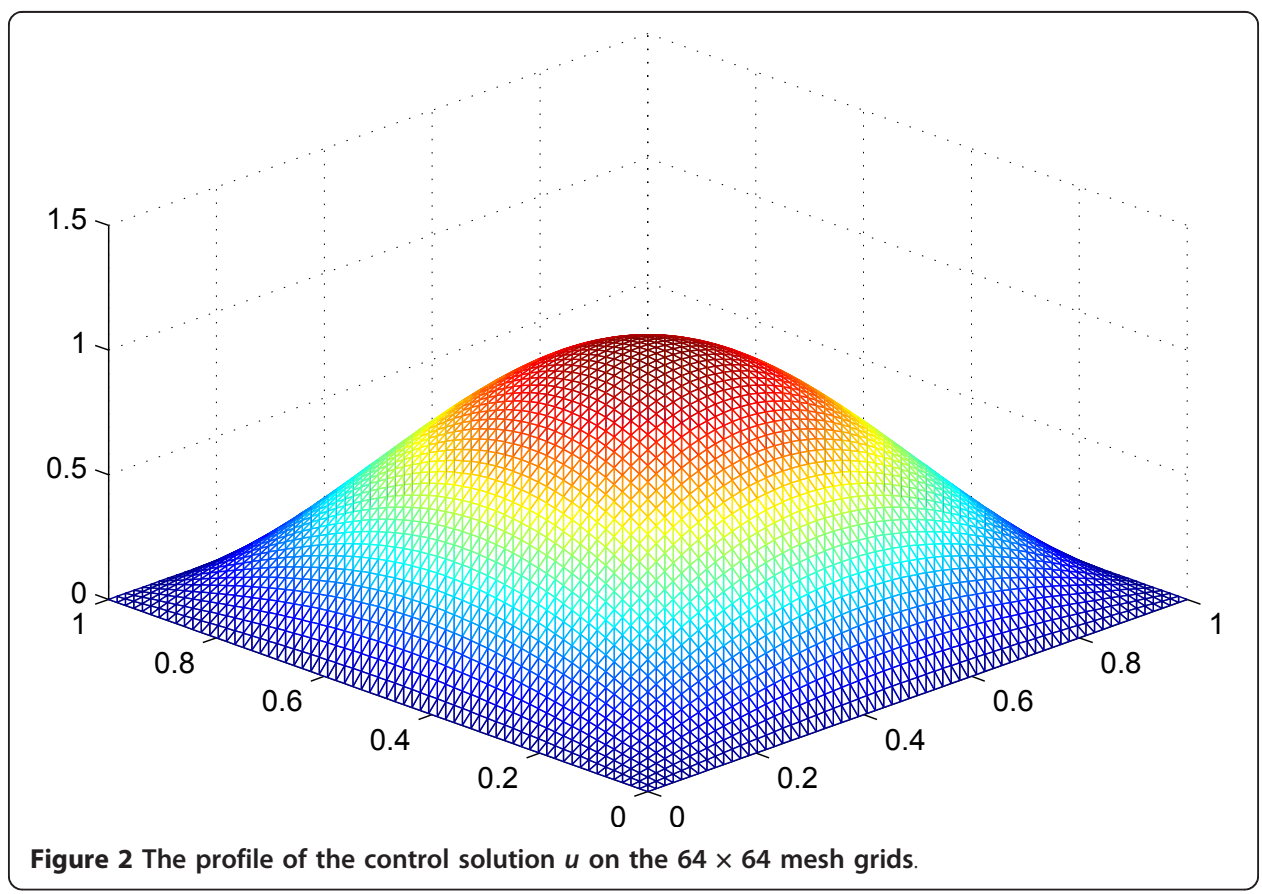


for the elliptic optimal control problems by variational discretization and mixed finite element methods seem to be new.

In our future study, we shall use the variational discretization and mixed finite element method to deal with the optimal control problems governed by nonlinear parabolic equations and convex boundary control problems. Furthermore, we shall consider a priori error estimates and superconvergence of optimal control problems governed by nonlinear parabolic equations or convex boundary control problems.

\section{Acknowledgements}

The authors express their thanks to the referees for their helpful suggestions, which led to improvements of the presentation. Zuliang Lu was by the supported National Science Foundation of China (11126329) and China Postdoctoral Science Foundation (2011M500968). Yanping Chen was supported by the Guangdong Province Universities and Colleges Pearl River Scholar Funded Scheme (2008), National Science Foundation of China (10971074), and Specialized Research Fund for the Doctoral Program of Higher Education (20114407110009). Yunqing Huang was supported by the NSFC Key Project (11031006) and Hunan Provincial NSF Project (10JJ7001).

\section{Author details}

${ }^{1}$ College of Civil Engineering and Mechanics, Xiangtan University, Xiangtan 411105, PR China ${ }^{2}$ School of Mathematics and Statistics, Chongqing Three Gorges University, Chongqing 404000, PR China ${ }^{3}$ School of Mathematical Sciences, South China Normal University, Guangzhou 510631, PR China ${ }^{4}$ Hunan Key Laboratory for Computation and Simulation in Science and Engineering, Department of Mathematics, Xiangtan University, Xiangtan 411105, Hunan, PR China

\section{Authors' contributions}

ZL carried out the molecular genetic studies, participated in the sequence alignment and drafted the manuscript. YC participated in the design of the study and performed the statistical analysis. YH conceived of the study, and participated in its design and coordination. All authors read and approved the final manuscript.

\section{Competing interests}

The authors declare that they have no competing interests.

Received: 11 January 2012 Accepted: 20 April 2012 Published: 20 April 2012

\section{References}

1. Lions, JL: Optimal Control of Systems Governed by Partial Differential Equtions. Springer, Berlin (1971)

2. Falk, FS: Approximation of a class of optimal control problems with order of convergence estimates. J Math Anal Appl. 44, 28-47 (1973)

3. Geveci, T: On the approximation of the solution of an optimal control problem governed by an elliptic equation. RAIRO Numer Anal. 13, 313-328 (1979)

4. Arada, N, Casas, E, Tröltzsch, F: Error estimates for the numerical approximation of a semilinear elliptic control problem. Comput Optim Appl. 23, 201-229 (2002)

5. Malanowski, K: Convergence of approximation vs.regularity of solutions for convex control constrained optimal control systems. Appl Math Optim. 34, 134-156 (1992)

6. Chen, Y: Superconvergence of optimal control problems by rectangular mixed finite element methods. Math Comput. 77, 1269-1291 (2008)

7. Chen, Y, Dai, L, Lu, Z: Superconvergence of quadratic optimal control problems by triangular mixed finite elements. Adv Appl Math Mech. 75, 881-898 (2009)

8. Chen, Y, Liu, WB: Error estimates and superconvergence of mixed finite elements for quadratic optimal control. Int J Numer Anal Model. 3, 311-321 (2006)

9. Chen, Y, Lu, Z: Error estimates for parabolic optimal control problem by fully discrete mixed finite element methods. Finite Elem Anal Des. 46, 957-965 (2010)

10. Chen, Y, Lu, Z: Error estimates of fully discrete mixed finite element methods for semilinear quadratic parabolic optimal control problems. Comput Methods Appl Mech Eng. 199, 1415-1423 (2010)

11. Lu, Z, Chen, Y, Zhang, H: A priori error estimates of mixed finite element methods for nonlinear quadratic optimal control problems. Lobachevskii J Math. 29, 164-174 (2008)

12. Lu, Z, Chen, Y: A posteriori error estimates of triangular mixed finite element methods for semilinear optimal control problems. Adv Appl Math Mech. 1, 242-256 (2009)

13. $L u, Z$, Chen, $Y: L^{\infty}$-error estimates of triangular mixed finite element methods for optimal control problem govern by semilinear elliptic equation. Numer Anal Appl. 12, 74-86 (2009)

14. Hinze, M: A variational discretization concept in control constrained optimization: the linear-quadratic case. J Comput Optim Appl. 30, 45-63 (2010)

15. Liu, WB, Yan, NN: A posteriori error estimates for distributed convex optimal control problems. Numer Math. 101, 1-27 (2005)

16. Liu, WB, Yan, NN: A posteriori error estimates for control problems governed by nonlinear elliptic equation. Adv Comput Math. 15, 285-309 (2001)

17. Grisvard, P: Elliptic Problems in Nonsmooth Domains. Pitman, London (1985)

18. Babuska, I, Strouboulis, T: The Finite Element Method and its Reliability. Oxford University press, Oxford (2001) 
19. Miliner, FA: Mixed finite element methods for quasilinear second-order elliptic problems. Math Comput. 44, 303-320 (1985)

20. Carstensen, C: A posteriori error estimate for the mixed finite element method. Math Comput. 66, 465-476 (1997)

21. Brezzi, F, Fortin, M: Mixed and Hybrid Finite Element Methods. Springer, Berlin (1991)

22. Douglas, J Jr, Roberts, JE: Global estimates for mixed finite element methods for second order elliptic problems. Numer Funct Anal Optim. 22, 953-972 (2001)

23. Li, R, Liu, WB:http://www.math.jju.edu.cn/matkw/afepack.html

doi:10.1186/1029-242X-2012-95

Cite this article as: Lu et al:: A priori error estimates for higher order variational discretization and mixed finite element methods of optimal control problems. Journal of Inequalities and Applications 2012 2012:95.

\section{Submit your manuscript to a SpringerOpen ${ }^{\circ}$} journal and benefit from:

- Convenient online submission

- Rigorous peer review

- Immediate publication on acceptance

- Open access: articles freely available online

- High visibility within the field

- Retaining the copyright to your article

Submit your next manuscript at $\boldsymbol{s p r i n g e r o p e n . c o m ~}$ 